\title{
Status of DTPA-Extractable Micronutrients in Valley Districts of Manipur
}

\author{
N. Surbala Devi*, R.K. Kumarjit Singh, Indira Sarangthem and T. Sanahanbi Devi \\ Department of Soil Science and Agricultural Chemistry, College of Agriculture, Central \\ Agricultural University, Imphal-795004, Manipur, India
}

*Corresponding author

\begin{tabular}{|c|c|}
\hline & A B S T R A \\
\hline & \multirow{6}{*}{ 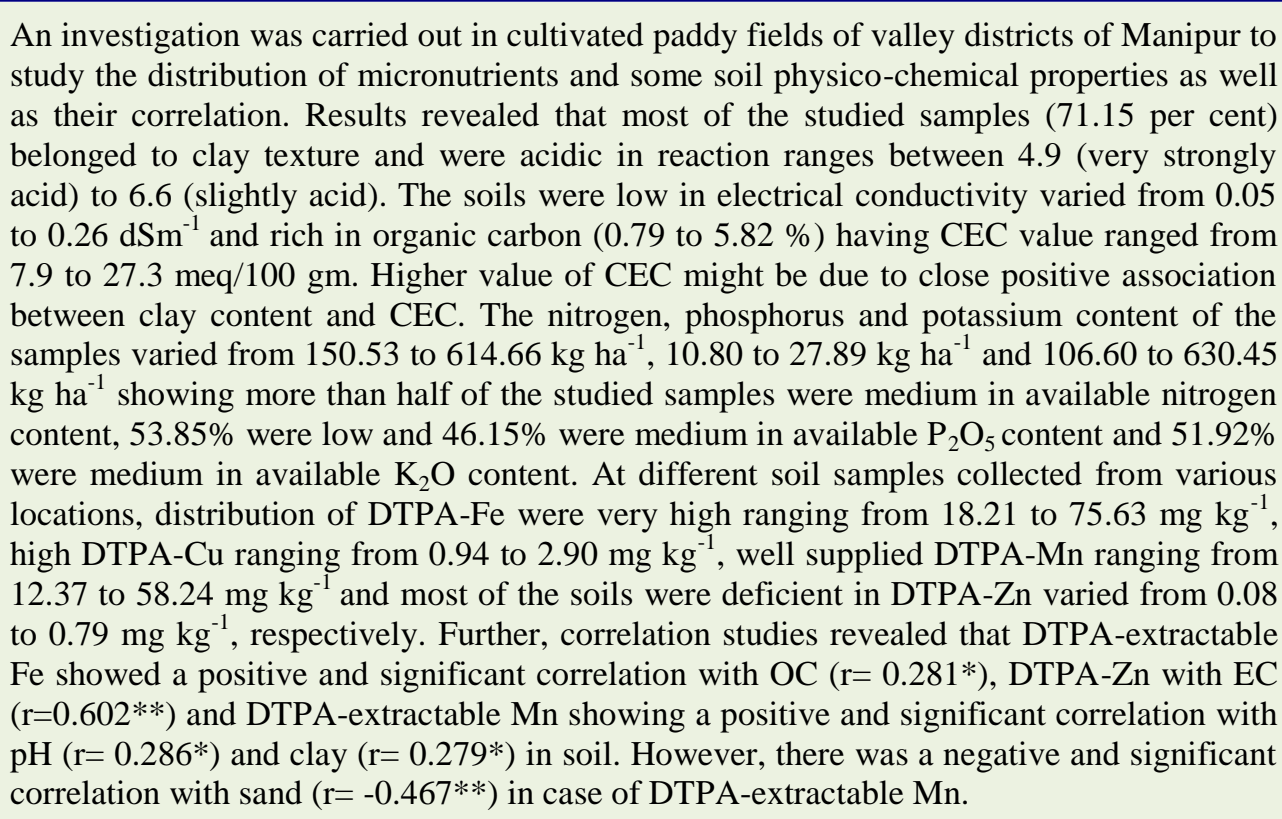 } \\
\hline Keywor & \\
\hline & \\
\hline Article Info & \\
\hline & \\
\hline & \\
\hline
\end{tabular}

\section{Introduction}

Indian soils have become deficient not only in major plant nutrients like nitrogen, phosphorus and in some cases, potash but also in secondary nutrients, like sulphur, calcium, and magnesium.

Micronutrients such as zinc, boron and to a limited extent iron, manganese, copper and molybdenum have also been reported to be deficient. Micronutrient deficiencies have become one of the major constraints in sustaining crop production in the present day exploitive agriculture. The importance of the micronutrients for different physiological processes in plant life is well understood, as they are required in smaller quantities. Therefore, when a nutrient becomes deficient in soil, the plant growth may suffer and the 
situation results into crop failure or loss of yield (Mengel et al., 2001; Gupta, 2003; Nazakat et al., 2012).

Micronutrients often act as cofactors in enzyme systems and participate in redox reactions, in addition to having several other vital functions in plants (Memon et al., 2012). Most importantly, these are involved in the key physiological processes of photosynthesis and respiration (Mengel et al., 2001; Gao et al., 2008) and their deficiency can impede the vital physiological processes thus limiting yield of the crop (Patil et al., 2008). For example, for rice (Oryza sativa L.), zinc deficiency is a major yield-limiting factor in several Asian countries (Wissuwa et al., 2006; Rehman et al., 2012). Deficiency of micronutrients has grown in both, magnitude and extent because of increased use of high analysis fertilizers, use of high yielding crop varieties and increase in cropping intensity which has become a major constraint to production and productivity of rice, wheat and pulses. The rice-wheat cropping system, being exhaustive in terms of nutrient removal, resulted in increased pressure on native nutrient reserve of soil. The deficiency of $\mathrm{Fe}$ and $\mathrm{Mn}$ has been found to occur in this cropping system (Takkar and Nayyar, 1981).

Availability of plant nutrients to crops has a strong bearing on physico-chemical nature of soils. India has a vast area under acid soils as well as sodic soils. Productivity of such soils can be restored through well-established ameliorative techniques. Use of lime or liming materials in acid soils and that of gypsum/phosphogypsum in sodic soils has been advocated by Soil Scientists for correction of soil $\mathrm{pH}$ and improving physicochemical nature of these soils. The availability of micronutrients is influenced by their distribution within the soil profile and other soil characteristics (Singh et al., 1989). The distribution of micronutrients may also differ among the profiles developed on different parent materials and landforms (Verma et al., 2005). Thus, there is an urgent need for correction of individual nutrient deficiency and for arresting its further spread.

Keeping these in view, the present study was undertaken to study the distribution of available micronutrients in paddy fields of valley districts of Manipur (India) as well as their correlation with some soil physicochemical properties.

\section{Materials and Methods}

The study area is a small plain covering an extent of $1843 \mathrm{sq} . \mathrm{km}$ in the central part of the state, known as Imphal valley and situated at about $24^{\circ} 44^{\prime} \mathrm{N}$ latitude and $93^{\circ} 58^{\prime}$ E longitude, at an altitude of about 790 metres above the mean sea level (MSL). The area falls under humid subtropical climate with mild, dry winters and a hot monsoon season having transported alluvium type of soil. The valley receives annual average rainfall of about $1,320 \mathrm{~mm}$, with June the wettest. Soil samples were collected from four valley districts viz., Imphal East, Imphal West, Thoubal and Bishnupur districts of Manipur (India) following stratified random sampling through proportional allocation. Total number of samples collected from different cultivated paddy fields of the four valley districts were 52 (Table 1).

The collected samples were processed and analyzed following standard procedures: for mechanical analysis using hydrometer method (Bouyoucous, 1927); soil pH and electrical conductivity (EC) using 1: 2.5 soil water suspensions (Jackson, 1973), CEC by leaching with $1 \mathrm{~N} \mathrm{NH}_{4} \mathrm{OAc}$ (Jackson, 1973) and organic carbon following Walkley and Black's rapid titration method (Walkley and Black, 1934). The samples were determined for available nitrogen (potassium permanganate method), 
available $\mathrm{P}_{2} \mathrm{O}_{5}$ (Bray and Kurtz No.1), neutral ammonium acetate extractable $\mathrm{K}_{2} \mathrm{O}$ and DTPA extractable $\mathrm{Zn}, \mathrm{Cu}, \mathrm{Mn}$ and $\mathrm{Fe}$ following standard procedures as outlined by Jackson (1973). The relationship between various soil properties and micro-nutrients distribution were established by using simple correlation coefficient.

\section{Results and Discussion}

\section{Soil texture}

Distribution of soil separates viz., sand, silt and clay in the soil samples are presented in Table 2. Result revealed that most of the studied samples (71.15per cent) belonged to clay texture. Higher clay content of the soils of Manipur was also reported by Sarker et al., (2002). However, all the remaining soils belonged to clay loam (3.85 per cent), silty clay loam (9.62 per cent), sandy clay (7.69 per cent), silty clay (5.77 per cent) or sandy loam (1.92 per cent). Sand, silt and clay fractions in the soils ranged from 06.8 to $55.9,05.0$ to 54.1 and 20.0 to 73.2 per cent, respectively.

Soils of Lanthrelloukon of Imphal East contained the highest amount of sand with a value of 55.9 per cent and the least was found in soils of Top Dasura, with a value of 06.8 per cent. The highest silt percentage was recorded in soils of Top Dasura (54.1 per cent) and the lowest in Takyel Mapal soils (05.0 per cent). Regarding clay content, the highest was found in soils of Nungoi (73.2 per cent) and lowest in Uyal soils (20.0 per cent).

\section{Soil reaction $(\mathrm{pH})$}

Soil $\mathrm{pH}$ (1:2.5, soil: water) values of the studied soil samples were, by and large, in the acidic range (Table 3). Most of the soil $\mathrm{pH}$ ranged between 4.9 (very strongly acid) to 6.6 (slightly acid). Nayak et al., (1996) and Sahoo et al., (2010) also reported similar acidic nature of the soils of Manipur. The acidity may be due to higher organic matter content (Nayak et al., 1996). $\mathrm{pH}$ value was found lowest in soils of Lalpani, Nachou and NgangkhaLawai and highest in Huikap with a value of 4.9 and 6.6 , respectively.

\section{Electrical Conductivity (EC)}

Data on soluble salt contents in the soil samples are presented in Table 3. Results revealed that electrical conductivity (EC) values (1:2.5, soil: water) varied from 0.05 to $0.26 \mathrm{dSm}^{-1}$. The lowest EC $\left(0.05 \mathrm{dSm}^{-1}\right)$ was observed in the soils of Champanagar, Kalika and Uyal and highest $\left(0.26 \mathrm{dSm}^{-1}\right)$ in Kangchup soil sample. Critical study of the data indicated that EC values were low $(<1$ $\left.\mathrm{dSm}^{-1}\right)$. This may be due to leaching loss of soluble salts from soils under high rainfall conditions (Brady and Weil, 1999 and Maji et al., 2005). On the basis of the limit suggested by Muhr et al., (1963) for judging salt problem, all the soil samples were found neutral $\left(\mathrm{EC}<1.0 \mathrm{dSm}^{-1}\right)$. The low EC of the soils may be due to low exchangeable bases. Similar observations were also reported by Sarkar et al., (2002).

\section{Organic carbon}

Data on organic carbon content of the studied samples (Table 3) revealed that its content in all the samples was high ranging from 0.79 to 5.82 per cent. The maximum organic carbon content was recorded in the soils of Thokchom with a value of 5.82 per cent and the lowest (0.79 per cent) in the soil samples of Hilgat and Dibong. Higher organic carbon content in the soils might be due to mixing of organic matter during cultivation as organic residues (Thangasamy et al., 2005). Sarkar et al., (2002) also reported higher organic carbon content in top layer soils of Manipur. Similar findings were also supported by Saha and Bala (1995) and Sahoo et al., (2010). 


\section{Cation Exchange Capacity (CEC)}

The cation exchange capacity (CEC) of the paddy field soils of valley districts of Manipur are presented in Table 3. Result revealed that CEC value ranged from 7.9 to $27.3 \mathrm{meq} / 100$ gm. Maximum soil samples (88.46 per cent) falls under medium range $(10-25 \mathrm{meq} / 100 \mathrm{~g})$. Higher value might be due to close positive association between clay content and CEC (Ghosh et al., 2005). On the other hand, it may perhaps be due to organic carbon in the surface layer. Further, it was also observed that soils of Lanthrel Loukon had the highest CEC with a value of $27.3 \mathrm{meq} / 100 \mathrm{gm}$ and the lowest was found in soils of Champanagar with a value of $7.9 \mathrm{meq} / 100 \mathrm{gm}$.

\section{Available Nitrogen (N)}

Data on available nitrogen content (Table 3) revealed that the soil samples contained available nitrogen varying from 150.53 to $614.66 \mathrm{~kg} \mathrm{ha}^{-1}$. The highest available nitrogen content $\left(614.66 \mathrm{~kg} \mathrm{ha}^{-1}\right)$ was observed in soils of Waiton and lowest in Hilgat and Nongren soils with a value of $150.53 \mathrm{~kg} \mathrm{ha}^{-1}$. The variation of available nitrogen content in the soils may be due to different amount of organic carbon present in the soils which released different amounts of nitrogen into the soils (Brady and Weil, 1999). On the basis of rating as suggested by Subbiah and Asija (1956) ( $<250 \mathrm{~N} \mathrm{~kg} \mathrm{ha}^{-1}$ for low, 250 to $500 \mathrm{~N}$ $\mathrm{kg} \mathrm{ha}^{-1}$ for medium and $>500 \mathrm{~N} \mathrm{~kg} \mathrm{ha}^{-1}$ for high), 36.54, 57.69 and 5.77 per cent soil samples fallen under low, medium and high categories, respectively. Detailed study revealed that more than half of the studied samples were medium in available nitrogen content.

\section{Available phosphorus $\left(\mathbf{P}_{2} \mathrm{O}_{5}\right)$}

Available phosphorus content in the soil varied from 10.80 to $27.89 \mathrm{~kg} \mathrm{ha}^{-1}$ (Table 3). It was observed that the highest available $\mathrm{P}_{2} \mathrm{O}_{5}$ content $\left(27.89 \mathrm{~kg} \mathrm{ha}^{-1}\right)$ was found in the soils of Uyal and lowest $\left(10.80 \mathrm{~kg} \mathrm{ha}^{-1}\right)$ in soil samples collected from Dibong and Hiyangthang.

On the basis of critical limit suggested by Muhr et al., (1963), all the soil samples were low. Higher available phosphorus content in surface layer might possibly be due to the confinement of crop cultivation to the rhizosphere and supplementing depleted phosphorus through external sources i.e., fertilizer and organic manure (Thangasamy et al., 2005 and Kumar et al., 2011).

This might be due to the fact that available $\mathrm{P}$ did mot leach out from the surface layer to the lower depth and is fixed by hydrated as well as amorphous oxide of $\mathrm{Fe}$ and $\mathrm{Al}$ which are potent phosphorus fixers in acidic soil (Brady and Weil, 1999).

Osodeke and Ubah (2005) also reported that at low $\mathrm{pH}$, the phosphorus may be fixed as $\mathrm{Fe}$ and $\mathrm{Al}$ phosphate, which are not likely to be readily available to plants.

\section{Available potassium $\left(\mathrm{K}_{2} \mathrm{O}\right)$}

Data on available potassium contents in different soil samples are presented in Table 3 . Results showed that available potassium content in different soil samples collected from various locations varied from 106.60 to $630.45 \mathrm{~kg} \mathrm{ha}^{-1}$. The highest available $\mathrm{K}$ content was recorded in soils of Ngangkha Lawai with a value of $630.45 \mathrm{~kg} \mathrm{ha}^{-1}$.

Higher available potassium content in soil surface could be attributed to release of labile $\mathrm{K}$ from organic residues and application of $\mathrm{K}$ fertilizers. Whereas, the lower potassium content in Hilgat soils (106.60 $\mathrm{kg} \mathrm{ha}^{-1}$ ) may be due to considerable leaching losses of soluble potassium due to high rainfall. 
Table.1 Locations of the soil samples collected

\begin{tabular}{|c|c|c|c|}
\hline District & Sub-divisions & Villages & No. of samples collected \\
\hline \multirow[t]{4}{*}{$\begin{array}{l}\text { IMPHAL EAST } \\
\text { (4 sub-divisions) }\end{array}$} & $\begin{array}{l}\text { 1.Jiribam } \\
\text { (Total villages- 51) }\end{array}$ & $\begin{array}{l}\text { 1. Hilgat } \\
\text { 2. Solapur } \\
\text { 3. Dibong } \\
\text { 4. Lalpani } \\
\text { 5. Champanagar }\end{array}$ & 5 \\
\hline & $\begin{array}{l}\text { 2. Sawombung } \\
\text { (Total villages- 64) }\end{array}$ & $\begin{array}{l}\text { 1. Tellou } \\
\text { 2. Tangkham } \\
\text { 3. Waiton } \\
\text { 4. Nungoi } \\
\text { 5. Kanglasagolmamg } \\
\text { 6. Pangei } \\
\text { 7. Nongren }\end{array}$ & 7 \\
\hline & $\begin{array}{l}\text { 3. KeiraoBitra } \\
\text { (Total villages- } 36 \text { ) }\end{array}$ & $\begin{array}{l}\text { 1. Kalika } \\
\text { 2. Huikap } \\
\text { 3. LanthrelLoukon } \\
\text { 4. Andro }\end{array}$ & 4 \\
\hline & $\begin{array}{l}\text { 4. Porompat } \\
\text { (Total villages- } 54 \text { ) }\end{array}$ & $\begin{array}{l}\text { 1. Top Dasura } \\
\text { 2. Wakha } \\
\text { 3. Gangapat } \\
\text { 4. Matai } \\
\text { 5. Khongman }\end{array}$ & 5 \\
\hline & & & TOTAL: 21 \\
\hline \multirow[t]{4}{*}{$\begin{array}{l}\text { IMPHAL WEST } \\
\text { (4 sub-divisions) }\end{array}$} & $\begin{array}{l}\text { 1. Lamshang } \\
\text { (Total villages- 69) }\end{array}$ & $\begin{array}{l}\text { 1. Kangchup } \\
\text { 2. AwangLeirenkabi } \\
\text { 3. Lamshang } \\
\text { 4. Phayeng } \\
\text { 5. Tharoijam }\end{array}$ & 5 \\
\hline & $\begin{array}{l}\text { 2. Patsoi } \\
\text { (Total villages- } 25 \text { ) }\end{array}$ & $\begin{array}{l}\text { 1. Yurembam } \\
\text { 2. Ghari } \\
\text { 3. Langjing }\end{array}$ & 3 \\
\hline & $\begin{array}{l}\text { 3. Lamphelpat } \\
\text { (Total villages- 9) }\end{array}$ & $\begin{array}{l}\text { 1. TakyelMapal } \\
\text { 2. MaibamLeikai }\end{array}$ & 2 \\
\hline & $\begin{array}{l}\text { 4. Wangoi } \\
\text { (Total villages- } 31 \text { ) }\end{array}$ & $\begin{array}{l}\text { 1. SangaiprouMamang } \\
\text { 2. Lairenjam } \\
\text { 3. Hiyangthang } \\
\text { 4. Monsangei }\end{array}$ & 4 \\
\hline & & & TOTAL: 14 \\
\hline \multirow[t]{3}{*}{$\begin{array}{l}\text { THOUBAL } \\
\text { (3 sub-divisions) }\end{array}$} & $\begin{array}{l}\text { 1. Thoubal } \\
\text { (Total villages- 49) }\end{array}$ & $\begin{array}{l}\text { 1. Charangpatmamang } \\
\text { 2. Uyal } \\
\text { 3. Bengi } \\
\text { 4. Thokchom }\end{array}$ & 4 \\
\hline & $\begin{array}{l}\text { 2. Lilong } \\
\text { (Total villages- } 17 \text { ) }\end{array}$ & $\begin{array}{l}\text { 1. Kekman } \\
\text { 2. Choubok } \\
\text { 3. Hoareibi }\end{array}$ & 3 \\
\hline & $\begin{array}{l}\text { 3. Kakching } \\
\text { (Total villages- } 37 \text { ) }\end{array}$ & $\begin{array}{l}\text { 1. KhakchingKhulel } \\
\text { 2. KakchingWairi } \\
\text { 3. Keirak } \\
\text { 4. Wabgai }\end{array}$ & 4 \\
\hline & & & TOTAL: 11 \\
\hline \multirow[t]{3}{*}{$\begin{array}{l}\text { BISHNUPUR } \\
\text { (3 sub-divisions) }\end{array}$} & $\begin{array}{l}\text { 1. Nambol } \\
\text { (Total villages- } 14 \text { ) }\end{array}$ & $\begin{array}{l}\text { 1. Utlou } \\
\text { 2. Keinou }\end{array}$ & 2 \\
\hline & $\begin{array}{l}\text { 2. Bishnupur } \\
\text { (Total villages- 13) }\end{array}$ & $\begin{array}{l}\text { 1. Nachou } \\
\text { 2. Potsangbam }\end{array}$ & 2 \\
\hline & $\begin{array}{l}\text { 3. Moirang } \\
\text { (Total villages- 22) }\end{array}$ & $\begin{array}{l}\text { 1. NgangkhaLawai } \\
\text { 2. Kumbi }\end{array}$ & 2 \\
\hline
\end{tabular}


Table.2 Particle size distribution of the soils collected from four valley districts of Manipur

\begin{tabular}{|c|c|c|c|c|c|}
\hline \multirow{2}{*}{$\begin{array}{c}\text { Sl. } \\
\text { No. } \\
\end{array}$} & \multirow[t]{2}{*}{ Villages } & \multicolumn{3}{|c|}{ Soil Separates } & \multirow[t]{2}{*}{ Soil Texture } \\
\hline & & Sand (\%) & Silt (\%) & Clay $(\%)$ & \\
\hline 1 & Hilgat & 27.3 & 43.6 & 29.1 & Clay loam \\
\hline 2 & Solapur & 21.8 & 17.5 & 60.7 & Clay \\
\hline 3 & Dibong & 22.7 & 35.7 & 41.6 & Clay \\
\hline 4 & Lalpani & 28.0 & 42.0 & 30.0 & Clay loam \\
\hline 5 & Champanagar & 19.3 & 42.5 & 38.2 & Silty clay loam \\
\hline 6 & Tellou & 14.3 & 20.0 & 65.7 & Clay \\
\hline 7 & Tangkham & 11.8 & 24.1 & 64.1 & Clay \\
\hline 8 & Waiton & 16.8 & 14.1 & 69.1 & Clay \\
\hline 9 & Nungoi & 16.8 & 10.0 & 73.2 & Clay \\
\hline 10 & Kanglasagolmamg & 14.3 & 22.5 & 63.2 & Clay \\
\hline 11 & Pangei & 11.8 & 22.5 & 65.7 & Clay \\
\hline 12 & Nongren & 16.8 & 29.1 & 54.1 & Clay \\
\hline 13 & Kalika & 21.8 & 31.6 & 46.6 & Clay \\
\hline 14 & Huikap & 21.8 & 15.0 & 63.2 & Clay \\
\hline 15 & LanthrelLoukon & 55.9 & 20.0 & 24.1 & Sandy clay \\
\hline 16 & Andro & 24.3 & 34.1 & 41.6 & Clay \\
\hline 17 & Top Dasura & 06.8 & 54.1 & 39.1 & Clay \\
\hline 18 & Wakha & 19.3 & 41.6 & 39.1 & Silty clay loam \\
\hline 19 & Gangapat & 11.0 & 42.0 & 47.0 & Silty clay \\
\hline 20 & Matai & 35.9 & 11.6 & 52.5 & Clay \\
\hline 21 & Khongman & 09.0 & 44.0 & 47.0 & Silty clay \\
\hline 22 & Kangchup & 35.9 & 10.0 & 54.1 & Clay \\
\hline 23 & AwangLeirenkabi & 38.4 & 13.2 & 48.4 & Clay \\
\hline 24 & Lamshang & 45.9 & 10.0 & 44.1 & Sandy clay \\
\hline 25 & Phayeng & 35.9 & 08.2 & 55.9 & Clay \\
\hline 26 & Tharoijam & 44.1 & 14.3 & 41.6 & Clay \\
\hline 27 & Yurembam & 38.0 & 19.0 & 43.0 & Clay \\
\hline 28 & Ghari & 30.7 & 25.2 & 44.1 & Clay \\
\hline 29 & Langjing & 35.9 & 11.6 & 52.5 & Clay \\
\hline 30 & TakyelMapal & 35.9 & 05.0 & 59.1 & Clay \\
\hline 31 & MaibamLeikai & 18.0 & 42.0 & 40.0 & Silty clay loam \\
\hline 32 & SangaiprouMamang & 19.0 & 42.0 & 39.0 & Silty clay loam \\
\hline 33 & Lairenjam & 32.5 & 10.9 & 56.6 & Clay \\
\hline 34 & Hiyangthang & 36.6 & 15.0 & 48.4 & Clay \\
\hline 35 & Monsangei & 35.9 & 10.0 & 54.1 & Clay \\
\hline 36 & Charangpatmamang & 53.4 & 11.4 & 35.2 & Sandy clay \\
\hline 37 & Uyal & 45.0 & 35.0 & 20.0 & Sandy loam \\
\hline 38 & Bengi & 15.0 & 22.0 & 63.0 & Clay \\
\hline 39 & Thokchom & 12.5 & 34.8 & 52.7 & Clay \\
\hline 40 & Kekman & 13.0 & 37.0 & 50.0 & Clay \\
\hline 41 & Choubok & 50.0 & 15.0 & 35.0 & Sandy clay \\
\hline 42 & Hoareibi & 11.6 & 30.0 & 58.4 & Clay \\
\hline 43 & KhakchingKhulel & 20.0 & 21.6 & 58.4 & Clay \\
\hline 44 & KakchingWairi & 13.9 & 23.0 & 64.1 & Clay \\
\hline 45 & Keirak & 14.1 & 21.8 & 64.1 & Clay \\
\hline 46 & Wabgai & 14.1 & 20.0 & 65.9 & Clay \\
\hline 47 & Utlou & 14.3 & 22.5 & 63.2 & Clay \\
\hline 48 & Keinou & 17.5 & 40.0 & 42.5 & Silty clay \\
\hline 49 & Nachou & 18.0 & 42.0 & 40.0 & Silty clay loam \\
\hline 50 & Potsangbam & 14.1 & 30.9 & 55.0 & Clay \\
\hline 51 & NgangkhaLawai & 20.0 & 25.0 & 55.0 & Clay \\
\hline 52 & Kumbi & 20.0 & 35.0 & 45.0 & Clay \\
\hline
\end{tabular}


Int.J.Curr.Microbiol.App.Sci (2018) 7(3): $3422-3433$

Table.3 Different chemical properties of the soils collected from four valley districts of Manipur

\begin{tabular}{|c|c|c|c|c|c|c|c|c|}
\hline Sl.no. & Villages & $\mathrm{pH}$ & $\begin{array}{c}\mathrm{EC}\left(\mathrm{dSm}^{-}\right. \\
1)\end{array}$ & $\begin{array}{l}\mathrm{OC} \\
(\%)\end{array}$ & $\begin{array}{c}\text { CEC (meq100 } \\
\text { gm }^{-1} \text { ) }\end{array}$ & $\begin{array}{c}\mathbf{N} \\
\left(\mathrm{kgha}^{-1}\right)\end{array}$ & $\begin{array}{c}\mathrm{P}_{2} \mathrm{O}_{5} \\
\left(\mathrm{kgha}^{-1}\right)\end{array}$ & $\begin{array}{c}\mathrm{K} 20 \\
\left(\mathrm{kgha}^{-1}\right)\end{array}$ \\
\hline 1 & Hilgat & 5.2 & 0.11 & 0.79 & 10.3 & 150.53 & 14.40 & 106.60 \\
\hline 2 & Solapur & 5.3 & 0.11 & 2.82 & 08.5 & 602.11 & 14.40 & 192.19 \\
\hline 3 & Dibong & 5.2 & 0.08 & 0.79 & 12.2 & 288.51 & 10.80 & 112.89 \\
\hline 4 & Lalpani & 4.9 & 0.15 & 2.11 & 10.5 & 589.57 & 11.80 & 129.02 \\
\hline 5 & Champanagar & 5.2 & 0.05 & 1.23 & 07.9 & 351.23 & 13.60 & 305.08 \\
\hline 6 & Tellou & 6.0 & 0.06 & 3.00 & 14.3 & 326.14 & 20.80 & 416.64 \\
\hline 7 & Tangkham & 5.2 & 0.17 & 2.55 & 13.0 & 288.51 & 27.00 & 158.59 \\
\hline 8 & Waiton & 5.9 & 0.11 & 3.70 & 16.0 & 614.66 & 17.40 & 563.13 \\
\hline 9 & Nungoi & 5.8 & 0.16 & 3.70 & 22.1 & 363.78 & 15.80 & 305.08 \\
\hline 10 & Kanglasagolmamg & 5.4 & 0.20 & 3.70 & 13.9 & 401.40 & 18.40 & 268.80 \\
\hline 11 & Pangei & 5.4 & 0.12 & 3.17 & 16.4 & 464.13 & 27.00 & 297.02 \\
\hline 12 & Nongren & 5.8 & 0.08 & 4.23 & 08.0 & 150.53 & 17.20 & 216.38 \\
\hline 13 & Kalika & 5.3 & 0.05 & 4.50 & 08.3 & 200.70 & 19.00 & 529.53 \\
\hline 14 & Huikap & 6.6 & 0.50 & 3.61 & 13.0 & 288.51 & 26.00 & 396.48 \\
\hline 15 & LanthrelLoukon & 5.5 & 0.11 & 4.41 & 27.3 & 225.79 & 26.00 & 606.14 \\
\hline 16 & Andro & 5.2 & 0.18 & 2.56 & 11.6 & 225.79 & 14.40 & 374.97 \\
\hline 17 & Top Dasura & 5.8 & 0.06 & 3.35 & 10.2 & 188.16 & 27.00 & 176.06 \\
\hline 18 & Wakha & 5.4 & 0.07 & 4.41 & 11.0 & 250.88 & 19.00 & 157.24 \\
\hline 19 & Gangapat & 5.7 & 0.08 & 3.00 & 11.2 & 213.25 & 17.20 & 384.38 \\
\hline 20 & Matai & 5.4 & 0.12 & 3.35 & 17.2 & 220.25 & 25.35 & 310.34 \\
\hline 21 & Khongman & 5.6 & 0.09 & 2.90 & 15.4 & 250.00 & 20.35 & 280.50 \\
\hline 22 & Kangchup & 5.0 & 0.26 & 2.64 & 09.4 & 200.70 & 27.00 & 147.84 \\
\hline 23 & AwangLeirenkabi & 5.1 & 0.14 & 3.26 & 11.8 & 263.42 & 26.00 & 353.47 \\
\hline 24 & Lamshang & 5.5 & 0.12 & 3.44 & 24.0 & 275.97 & 17.20 & 275.52 \\
\hline 25 & Phayeng & 5.1 & 0.19 & 2.38 & 10.3 & 225.79 & 25.40 & 176.06 \\
\hline 26 & Tharoijam & 5.2 & 0.08 & 4.32 & 10.6 & 275.97 & 19.60 & 153.21 \\
\hline 27 & Yurembam & 5.2 & 0.12 & 3.17 & 10.8 & 275.97 & 27.00 & 397.82 \\
\hline 28 & Ghari & 5.3 & 0.10 & 4.14 & 10.7 & 351.23 & 15.00 & 196.22 \\
\hline 29 & Langjing & 5.4 & 0.09 & 4.05 & 12.6 & 301.06 & 27.00 & 211.00 \\
\hline 30 & TakyelMapal & 5.2 & 0.12 & 4.14 & 22.2 & 275.97 & 11.32 & 241.92 \\
\hline 31 & MaibamLeikai & 5.6 & 0.14 & 4.50 & 21.2 & 280.00 & 15.49 & 300.10 \\
\hline 32 & SangaiprouMamang & 5.3 & 0.12 & 4.41 & 19.1 & 263.42 & 27.00 & 400.51 \\
\hline 33 & Lairenjam & 5.0 & 0.11 & 4.50 & 22.0 & 250.88 & 27.00 & 298.36 \\
\hline 34 & Hiyangthang & 5.2 & 0.22 & 4.23 & 24.0 & 338.69 & 10.80 & 188.16 \\
\hline 35 & Monsangei & 5.4 & 0.08 & 4.14 & 21.0 & 188.16 & 27.00 & 245.95 \\
\hline 36 & Charangpatmamang & 5.3 & 0.08 & 3.79 & 23.1 & 225.79 & 15.60 & 198.91 \\
\hline 37 & Uyal & 5.3 & 0.05 & 4.41 & 21.4 & 213.25 & 27.89 & 211.00 \\
\hline 38 & Bengi & 5.4 & 0.09 & 3.89 & 23.6 & 238.33 & 27.00 & 209.66 \\
\hline 39 & Thokchom & 5.0 & 0.13 & 5.82 & 19.9 & 275.97 & 27.00 & 280.90 \\
\hline 40 & Kekman & 5.7 & 0.06 & 4.41 & 10.3 & 225.79 & 26.89 & 229.82 \\
\hline 41 & Choubok & 5.8 & 0.06 & 4.58 & 12.0 & 250.88 & 14.80 & 232.51 \\
\hline 42 & Hoareibi & 5.5 & 0.16 & 5.64 & 17.0 & 175.61 & 16.80 & 341.38 \\
\hline 43 & KhakchingKhulel & 5.4 & 0.08 & 5.38 & 23.1 & 225.79 & 14.40 & 395.13 \\
\hline 44 & KakchingWairi & 5.4 & 0.11 & 5.82 & 23.7 & 301.06 & 14.40 & 471.74 \\
\hline 45 & Keirak & 5.1 & 0.11 & 5.02 & 21.0 & 338.69 & 26.89 & 423.36 \\
\hline 46 & Wabgai & 5.4 & 0.08 & 5.73 & 18.0 & 288.51 & 27.00 & 202.94 \\
\hline 47 & Utlou & 5.4 & 0.11 & 2.71 & 21.4 & 163.07 & 25.45 & 554.96 \\
\hline 48 & Keinou & 5.5 & 0.13 & 3.00 & 19.3 & 192.00 & 18.45 & 450.45 \\
\hline 49 & Nachou & 4.9 & 0.08 & 3.92 & 21.2 & 350.50 & 17.90 & 510.45 \\
\hline 50 & Potsangbam & 5.1 & 0.09 & 4.29 & 15.6 & 373.20 & 16.40 & 490.00 \\
\hline 51 & NgangkhaLawai & 4.9 & 0.08 & 4.84 & 23.5 & 345.00 & 18.45 & 630.45 \\
\hline 52 & Kumbi & 5.0 & 0.08 & 4.26 & 23.2 & 290.80 & 21.10 & 520.20 \\
\hline
\end{tabular}


Table.4 Distribution of micronutrients of the soils collected from four valley districts of Manipur

\begin{tabular}{|c|c|c|c|c|c|}
\hline SI No. & Villages & $\begin{array}{c}\mathrm{Fe} \\
\left(\mathrm{mg} \mathrm{kg}^{-1}\right)\end{array}$ & $\begin{array}{c}\mathrm{Mn} \\
\left(\mathrm{mg} \mathrm{kg}^{-1}\right)\end{array}$ & $\begin{array}{c}\mathrm{Cu} \\
\left(\mathrm{mg} \mathrm{kg}^{-1}\right)\end{array}$ & $\begin{array}{c}\mathrm{Zn}_{(\mathbf{m g ~ k g}} \\
(\mathbf{m g ~})\end{array}$ \\
\hline 1 & Hilgat & 26.75 & 43.94 & 0.94 & 0.15 \\
\hline 2 & Solapur & 38.87 & 12.37 & 1.63 & 0.46 \\
\hline 3 & Dibong & 27.11 & 36.78 & 1.9 & 0.26 \\
\hline 4 & Lalpani & 28.03 & 18.46 & 1.85 & 0.45 \\
\hline 5 & Champanagar & 35.05 & 34.26 & 1.3 & 0.32 \\
\hline 6 & Tellou & 44.44 & 53.34 & 2.6 & 0.11 \\
\hline 7 & Tangkham & 70.01 & 44.83 & 2.9 & 0.3 \\
\hline 8 & Waiton & 50.84 & 41.32 & 1.84 & 0.16 \\
\hline 9 & Nungoi & 47.82 & 58.24 & 1.91 & 0.12 \\
\hline 10 & Kanglasagolmamg & 58.93 & 45.47 & 2.06 & 0.18 \\
\hline 11 & Pangei & 50.95 & 32.43 & 1.26 & 0.12 \\
\hline 12 & Nongren & 36.72 & 55.95 & 1.78 & 0.18 \\
\hline 13 & Kalika & 52.58 & 51.97 & 2.36 & 0.25 \\
\hline 14 & Huikap & 18.21 & 21.36 & 1.15 & 0.79 \\
\hline 15 & LanthrelLoukon & 52.29 & 21.59 & 1.94 & 0.41 \\
\hline 16 & Andro & 68.83 & 35.12 & 1.19 & 0.51 \\
\hline 17 & Top Dasura & 31.94 & 42.04 & 1.73 & 0.08 \\
\hline 18 & Wakha & 56.67 & 19.3 & 1.7 & 0.16 \\
\hline 19 & Gangapat & 44.45 & 34.52 & 1.75 & 0.18 \\
\hline 20 & Matai & 32.45 & 21.45 & 1.81 & 0.1 \\
\hline 21 & Khongman & 44.56 & 39.8 & 1.89 & 0.15 \\
\hline 22 & Kangchup & 54.47 & 27.26 & 2.21 & 0.39 \\
\hline 23 & AwangLeirenkabi & 64.25 & 17.91 & 2.51 & 0.43 \\
\hline 24 & Lamshang & 42.21 & 31.96 & 2.06 & 0.42 \\
\hline 25 & Phayeng & 49.62 & 17.72 & 2.25 & 0.32 \\
\hline 26 & Tharoijam & 47.53 & 15.35 & 2.06 & 0.13 \\
\hline 27 & Yurembam & 44.81 & 30.23 & 1.46 & 0.2 \\
\hline 28 & Ghari & 37.6 & 12.94 & 1.36 & 0.13 \\
\hline 29 & Langjing & 45.88 & 24.17 & 2.26 & 0.3 \\
\hline 30 & TakyelMapal & 45.67 & 25.76 & 1.5 & 0.14 \\
\hline 31 & MaibamLeikai & 37.65 & 23.24 & 1.36 & 0.09 \\
\hline 32 & SangaiprouMamang & 50.04 & 18.51 & 1.62 & 0.18 \\
\hline 33 & Lairenjam & 66.15 & 13.88 & 1.84 & 0.11 \\
\hline 34 & Hiyangthang & 57.32 & 22.99 & 2.22 & 0.28 \\
\hline 35 & Monsangei & 29.79 & 15.4 & 1.27 & 0.08 \\
\hline 36 & Charangpatmamang & 67.2 & 28.63 & 1.81 & 0.28 \\
\hline 37 & Uyal & 49.53 & 29.4 & 1.5 & 0.08 \\
\hline 38 & Bengi & 44.88 & 44.05 & 1.67 & 0.12 \\
\hline 39 & Thokchom & 52.55 & 30.07 & 1.68 & 0.2 \\
\hline 40 & Kekman & 75.63 & 29.39 & 2.38 & 0.43 \\
\hline 41 & Choubok & 53.1 & 24.16 & 2.08 & 0.18 \\
\hline 42 & Hoareibi & 41.86 & 41.75 & 1.82 & 0.16 \\
\hline 43 & KhakchingKhulel & 38.41 & 40.85 & 2.05 & 0.26 \\
\hline 44 & KakchingWairi & 51.69 & 27.57 & 1.75 & 0.18 \\
\hline 45 & Keirak & 57.59 & 28.08 & 1.87 & 0.15 \\
\hline 46 & Wabgai & 54.29 & 29.33 & 1.76 & 0.13 \\
\hline 47 & Utlou & 51.69 & 24.63 & 1.87 & 0.18 \\
\hline 48 & Keinou & 50.63 & 23.11 & 1.91 & 0.14 \\
\hline 49 & Nachou & 49.7 & 26.73 & 2.3 & 0.21 \\
\hline 50 & Potsangbam & 50.01 & 35.2 & 1.99 & 0.18 \\
\hline 51 & NgangkhaLawai & 47.34 & 34.84 & 2.03 & 0.22 \\
\hline 52 & Kumbi & 43.42 & 32.10 & 2.05 & 0.25 \\
\hline
\end{tabular}


Table.5 Correlation coefficient ( $\mathrm{r}$ ) between DTPA - extractable micronutrients and physicochemical properties of soils

\begin{tabular}{|c|c|c|c|c|}
\hline \multirow[t]{2}{*}{ Soil Property } & \multicolumn{4}{|c|}{ DTPA - extractable micronutrients } \\
\hline & $\mathrm{Fe}$ & $\mathrm{Mn}$ & $\mathrm{Cu}$ & $\mathrm{Zn}$ \\
\hline Sand & 0.053 & $-0.467 * *$ & 0.011 & 0.200 \\
\hline Silt & -0.170 & 0.200 & -0.202 & -0.135 \\
\hline Clay & 0.120 & $0.279 *$ & 0.194 & -0.070 \\
\hline pH & -0.264 & $0.286^{*}$ & -0.129 & 0.110 \\
\hline EC & -0.133 & -0.147 & -0.126 & $0.602 * *$ \\
\hline CEC & 0.143 & -0.074 & -0.003 & -0.240 \\
\hline $\mathrm{OC}$ & $0.281 *$ & -0.088 & 0.106 & -0.249 \\
\hline $\mathbf{N}$ & -0.060 & -0.103 & 0.011 & 0.156 \\
\hline $\mathrm{P}_{2} \mathrm{O}_{5}$ & 0.228 & -0.151 & 0.121 & -0.034 \\
\hline $\mathbf{K}_{2} \mathbf{O}$ & 0.117 & 0.118 & 0.108 & 0.020 \\
\hline
\end{tabular}

$*$ Critical value of $\mathrm{r}$ significant at $5 \%$ and $* *$ at $1 \%$

\section{DTPA-extractable iron (Fe)}

The DTPA-extractable Fe content of the soils were fairly high in all the studied soil samples with a value varying from 18.21 to $75.63 \mathrm{mg}$ $\mathrm{kg}^{-1}$ (Table 4). The highest concentration of available Fe was found in the soils of Kekman and least in Huikap soils with a value of (75.63 $\mathrm{mg} \mathrm{kg}^{-1}$ ) and (18.21 $\left.\mathrm{mg} \mathrm{kg}^{-1}\right)$, respectively. Considering critical limit of 4.5 mg Fe kg ${ }^{-1}$ soil (Lindsay and Norvell, 1978) all the soils were highly sufficient in available Fe. This may be attributed to higher organic matter because it acts as chelating agent (Kumar et al., 2011).

These cationic micronutrients react with certain organic molecules to form organo metallic complexes as chelates, and the soluble chelates can increase the availability of the micronutrient and protect it from precipitation reactions. These chelates may also be synthesized by plant roots and released to the surrounding soil or may be present in soil humus (Brady and Weil, 1999).

Correlation studies revealed (Table 5) that DTPA-extractable Fe showed a positive and significant correlation with OC $\left(\mathrm{r}=0.281^{*}\right)$ in soil. The result was in conformity with the findings of Sarkar et al., (2000); Sharma et al., (2002) and Verma et al., (2007).

\section{DTPA-extractable manganese (Mn)}

Data on distribution of DTPA-extractable Mn are shown in Table 4. DTPA-Mn content ranged from 12.37 to $58.24 \mathrm{mg} \mathrm{kg}^{-1}$ soils in soil with mean value of $30.61 \mathrm{mg} \mathrm{kg}^{-1}$. The highest content of available Mn was found in soil of Nungoi village and lowest in Solapur village of Imphal East district with a value of 58.24 and $12.37 \mathrm{mg} \mathrm{kg}^{-1}$ soil, respectively. Considering $1.0 \mathrm{mg} \mathrm{Mn} \mathrm{kg}{ }^{-1}$ soil as critical level (Lindsay and Norvell, 1978), all the samples were well supplied with available Mn. The abundance of DTPA - Mn in soils of Manipur was also reported by Sarkar et al., (2002).

Correlation studies (Table 5) revealed that DTPA-extractable Mn showed a positive and significant correlation with $\mathrm{pH}\left(\mathrm{r}=0.286^{*}\right)$ and clay $\left(r=0.279^{*}\right)$. Similar observations were also given by Singh et al., (2006) and Tiwary and Mishra (1990) in soil. However, there was a negative and significant correlation with sand $(\mathrm{r}=-0.467 * *)$. 


\section{DTPA-extractable copper $(\mathrm{Cu})$}

Results (Table 4) revealed that DTPAextractable $\mathrm{Cu}$ content in different soil samples ranged from 0.94 to $2.90 \mathrm{mg} \mathrm{kg}^{-1}$ soil. It was also observed that soil of Tangkham village showed higher concentration $\left(2.90 \mathrm{mg} \mathrm{kg}^{-1}\right)$ of available $\mathrm{Cu}$ and the lowest $\left(0.94 \mathrm{mg} \mathrm{kg}^{-1}\right)$ in soil of Hilgat village. Maximum DTPA- extractable $\mathrm{Cu}$ recorded in these surface layer soil are in close proximity with the findings of Sharma et al., (2002); Sharma et al., (2005) and Athokpam et al., (2013).

Taking into consideration the critical value of $0.20 \mathrm{mg} \mathrm{Cu} \mathrm{kg}^{-1}$ (Lindsay and Norvell, 1978), all the soil samples were well supplied with available $\mathrm{Cu}$. Similar findings were also made by Sen et al., (1997).

\section{DTPA-extractable Zinc (Zn)}

DTPA-extractable Zn content in soil samples were analysed (Table 4) and indicated that DTPA- $\mathrm{Zn}$ content varied from 0.08 to 0.79 $\mathrm{mg} \mathrm{kg}{ }^{-1}$ soil. Among the different soil samples, soils collected from Huikap showed the highest DTPA- Zn content $\left(0.79 \mathrm{mg} \mathrm{kg}^{-1}\right)$ and least in soils of Top Dasura, Monsangei and Uyal with a value of $0.08 \mathrm{mg} \mathrm{kg}^{-1}$. Based on critical limit of $0.6 \mathrm{mg} \mathrm{kg}^{-1}$ soil (Lindsay and Norvell, 1978), most of the soils are deficient in $\mathrm{Zn}$ content and required $\mathrm{Zn}$ fertilization for better crop production. Sen et al., (1997) and Sarkar et al., (2002) also observed $\mathrm{Zn}$ deficiency in soils of Manipur.

Correlation studies in Table 5 showed that DTPA-Zn was positively and significantly correlated with EC $(\mathrm{r}=0.602 * *)$. The result was in conformity with the findings of Athokpam et al., (2013).

The study thus indicated that the acidity of the soil may be due to higher organic matter content in soil and leaching loss of soluble salts from soils under high rainfall conditions causing low EC. Higher value of CEC might be due to close positive association between clay content and CEC and also organic carbon in the surface layer. The samples were well supplied with available $\mathrm{Fe}, \mathrm{Cu}$ and $\mathrm{Mn}$. The deficiency of $\mathrm{Zn}$ is of major concern among all the micronutrients. Organic carbonhad a prominent effect on the availability of micronutrients. Micronutrients are very important for maintaining soil health and also in increasing productivity of crops. Keeping in view the low content of $\mathrm{Zn}$ in soil, $\mathrm{Zn}$ fertilization is suggested in order to improve the nutritional status of soil and to ensure optimum crop production.

\section{References}

Athokpam, H., Wani, S.K., Kamei, D., Athokpam, H.S., Nongmaithem, J., Kumar, D., Singh, Y.K., Naorem, B.S., Devi, T.R. and Devi, L. (2013). Soil macro- and micro-nutrient status of senapati district, Manipur. African Journal of Agricultural Research 8(39):4932-4936.

Gao, S., Yan, R., Cao, M., Yang, W., Wang, S. and Chen, F. (2008). Effect of copper on growth, antioxidant enzymes and phenyalanine ammonia-lyse activities in Jatrophacurcas I., seedling. Plant, Soil and Environment. 54:117-122.

Ghosh, S., Sarkar, D. and Sahoo, A.K. (2005). Nature, Distirbution and Amelioration response of soil acidity in a host site of hot dry sub-humic belt of West Bengal. Journal of the Indian Society of Soil Science 53(1): 66-73.

Gupta, P.K. (2003). A Hand Book of Soil, Fertilizer and manure (2nd Edn.).

Kasraei, R., Barrueco, C.R. and Arroyo, M.I. (1996). The effect of $\mathrm{Al}$ and $\mathrm{Mn}$ on growth and mineral composition of Casuarinaequisetifolia Forst. In: C. 
Rodriguez Barrueco (ed.), Fertilizers and Environment, Kluwer Academic Publishers, Printed in the Netherlands, pp. 75-81.

Kumar, M., Singh, S.K., Raina, P. and Sharma, B.K. (2011). Status of available major and micronutrients in arid soils of Churu District of Western Rajasthan. Journal of the Indian Society of Soil Science. 52(2): 188-192.

Memon, M., Jamro, G.M., Memon, N.N., Memon, K.S. and Akhtar, M.S. (2012). Micronutrient availability assessment of Tomato grown in taluka Badin, Sindh. Pakistan journal of Botany. 44(2): 649654.

Mengel, K., Kirkby, E.A., Kosegarten, H. and Appel, T. (2001). Principles of Plant Nutrition. Kluwer Academic Publishers, Dordrecht, The Netherlands.

Muhr, G.R., Datta, N.P., Shankara, S.N., Dever, E., Lecy, V.K. and Dovahue, R.R. (1963). Soil Testing in Indian USAID Mission to India.

Nayak, D.C., Sen, T.K., Chamuah, G.S. and Sehgal, J.L. (1996). Nature of soil acidity in some soils of Manipur. Journal of the Indian Society of Soil Science. 44: 209-214.

Nazakat, N., Nawaz, M.S., Cheema, N.M. and Khan, M.A. (2012). Zinc and Iron application to optimized seed yield of Mustard. Pakistan Journal Agricultural Research.25 (1): 28-33.

Patil, B.C., Hosamani, R.M., Ajjappalavara, P.S., Naik, B.H., Smitha, R.P. and Ukkund, K.C. (2008). Effect of foliar application of micronutrients on growth and yield components of tomato (Lycopersicon esculentum Mill.). Karnataka. Journal Agricultural Sciences. 21(3): 428-430.

Rehman, H., Aziz, T., Farooq, M., Wakeel, A. and Rengel, Z. (2012). Zinc nutrition in rice production systems. Plant and Soil. 361: 203-226.
Saha, G.C. and Bala, N. (1995). Characterization and classification of soils on Valley Plains of Middle Andaman Island. Journal of the Indian Society of Soil Science. 43(1): 99-103.

Sahoo, A.K., Sarkar, D., Baruah, U. and Butte, P.S. (2010). Characterization, Classification and Evaluation of Soils of Langol Hill, Manipur for Rational Land Use Planning. Journal of the Indian Society of Soil Science 58(4): 355-362.

Sarkar, D., Baruah, V., Gangopadhyay, S.K., Sahoo, A.K. and Velayutham, M. (2002). Characteristics and classification of soils of Loktak command area of Manipur for sustainable land use planning. Journal of the Indian Society of Soil Science 50: 196-204.

Sarkar, D., Halder, A., Majumdar, A. and Velayutham, M. (2000). Distribution of micronutrient cations in some lnceptisols and Entisols of Madhubani Districts, Bihar. Journal of the Indian Society of Soil Science. 48(1): 202-205.

Sen, T.K., Dubey, P.N., Maji, A.K. and Chamuah, G.S. (1997). Status of micronutrient in some dominant soils of Manipur. Journal of the Indian Society of Soil Science. 45(2): 388-390.

Sharma, B.D., Aggarwal, V.K., Mukhopadhyay, S.S. and Arora, H. (2002). Micronutrient distribution and their association with soil properties in Entisols of Punjab. Indian Journal of Agricultural Sciences. 72(6): 334-340.

Sharma, B.D; Mukhopadhyay, S.S, and Arora, H. (2005). Total and DPTAextractable micronutrients in relation to pedogenesis in some Alfisols of Punjab. Journal of the Indian Society of Soil Sciences. 170(7): 559-572.

Singh, Kuldeep, Kuhad, M.S and Dhankar, S.S. (1989). Influence of soil characteristics on profile distribution of DTPA-extractable micronutrient 
cations. Indian Journal of Agricultural Sciences.59: 310-312.

Singh, R.D., Kumar, S. and Pande, H. (2006). Micronutrient status of soils under different vegetations in Uttaranchal hills. Journal of the Indian Society of Soil Science. 54(1): 115-116.

Subbiah B.V. and Asija G.L. (1956). A rapid procedure for estimation of available $\mathrm{N}$ in soils. Current Science. 25: 259-260.

Takkar, P. N. and Nayyar, Y. K. (1981). Preliminary field observation of Manganese deficiency in wheat and berseem. Fertiliser News.2: 22-23 and 33.

Thangasamy, A., Naidu, M.V.S., Ramavatharam. N. and Reddy, C.R. (2005). Characterization, classification and evaluation of soil resources in Sivagiri micro-watershed of Chittoor district in Andhra Pradesh for sustainable land used planning. Journal of the Indian Society of Soil Science. 53(1): 515-520.
Tiwary, J.R. and Mishra, B.B. (1990). Distribution of micronutrients in Tal land soils (Udic Chromusterts) of Bihar. Journal of the Indian Society of Soil Science. 38(2): 319-321.

Verma, V.K., Setia, R.K., Sharma, P.K., Khurana, M.P.S. and Kang, S. (2007). Pedospheric distribution of micronutrient cations in soil developed on various landforms in north-east Punjab. Journal of the Indian Society of Soil Science. 55: 515-520.

Verma, Y.K., Setia, R.K., Sharma, P.K., Singh, C. and Kumar, A. (2005). Pedospheric variations in distribution of DTPA-extractable micronutrients in soils developed on different physiographic units in central parts of Punjab, India. International Journal of Agriculture and Biology. 2(2): 243-246.

Wissuwa, M., Ismail, A.M. and Yanagihara, S. (2006). Effects of zinc deficiency on rice growth and genetic factors contributing to tolerance. Plant Physiology.142: 731-741.

\section{How to cite this article:}

Surbala Devi, N., R.K. Kumarjit Singh, Indira Sarangthem and Sanahanbi Devi, T. 2018. Status of DTPA-Extractable Micronutrients in Valley Districts of Manipur. Int.J.Curr.Microbiol.App.Sci. 7(03): 3422-3433. doi: https://doi.org/10.20546/ijcmas.2018.703.394 